\title{
Los Retos de una Educación Virtual para Estudiantes con Necesidades Educativas Especiales
}

\section{The Challenges of a Virtual Education for Students with Special Educational Needs}

\author{
Milagros Esperanza Rojas Salgado ${ }^{1}$ \\ https://orcid.org/0000-0002-5750-0669 \\ Universidad Nacional Mayor de San Marcos, Perú
}

Recibido:14-01-2021

Aceptado: 18-04-2021

\section{Cita Recomendada}

Rojas, M.. (2021). Los retos de una educación virtual para estudiantes con necesidades educativas especiales. Hamut'ay, 8 (1), 9-22. http://dx.doi.org/10.21503/hamu.v8i1.2232

\begin{abstract}
Resumen
El presente artículo tiene como propósito identificar información relevante en relación con la experiencia de las madres, padres de familia o tutores acerca del proceso educativo en la modalidad no presencial, mediado por la tecnología, del que participan sus hijos, hijas o tutorados con Necesidades Educativas Especiales (NEE), así como explorar los retos que demanda la implementación de la educación en esta modalidad para estudiantes con NEE, teniendo en cuenta que, dicha implementación surge como respuesta a las demandas planteadas por un contexto de pandemia por la COVID - 19 en este sentido, el retorno a la presencialidad se visualiza como incierto y diferente a las condiciones educativas antes de esta pandemia. Como hallazgos se encontraron la necesidad de rediseñar un contexto educativo atendiendo al Diseño Universal de Aprendizaje y, en el que la interacción estudiante - docente se vea básicamente mediada no solo por las Tecnologías de la Información y la Comunicación (TIC), sino que también, el proceso educativo se sostenga en el establecimiento de sólidas relaciones de colaboración entre los docentes, los especialistas y los familiares del estudiante. A partir del presente estudio, se concluye que la asesoría contínua y especializada a las familias, la formación docente - no solo respecto del uso de la tecnología sino de la planificación de procesos de aprendizajes orientados a la atención de la diversidad-, además de aspectos de cobertura de TIC, son fundamentales para proponer la educación en la modalidad virtual para los estudiantes con NEE.
\end{abstract}

Palabras Clave: Necesidades Educativas Especiales, enseñanza, aprendizaje, tecnología.

1. Doctorando en Psicología de la Universidad Nacional Mayor de San Marcos. Egresada del Doctorado en Educación. Magíster en Gestión de la Educación. Licenciada en Psicología Humana y en Educación en la especialidad de Biología y Química. Estudios de posgrado en Neuropedagogía, Atención a la diversidad, Intervención con Personas de Educación Especial, TIC y enseñanza de las Ciencias Naturales. 


\begin{abstract}
The purpose of this article is to identify relevant information in relation to the experience of mothers, fathers or guardians about the educational process in the non-face-to-face modality, mediated by technology, in which their sons, daughters or guardians with Special Educational Needs (SEN) participate, as well as to explore the challenges that the implementation of education in this modality for students with SEN demands, taking into account that such implementation arises in response to the demands posed by a context of pandemic by the COVID - 19. In this context, the return to face-to-face education is seen as uncertain and different from the educational conditions before this pandemic. As findings, it was found the need to redesign an educational context according to the Universal Design for Learning and in which the student-teacher interaction is basically mediated not only by the Information and Communication Technologies (ICT), but also, the educational process is sustained in the establishment of strong collaborative relationships between teachers, specialists and the student's family members. From the present study, it is concluded that continuous and specialized advice to families, teacher training -not only in the use of technology but also in the planning of learning processes oriented to the attention of diversity-, as well as aspects of ICT coverage, are fundamental to propose education in the virtual modality for students with SEN.
\end{abstract}

Key words: Special Educational Needs, teaching, learning, technology.

\section{Introducción}

Desde fines del siglo XX las Tecnologías de la Información y la Comunicación (TIC) han desempeñado un papel fundamental en la construcción de la llamada Sociedad del Conocimiento, dado que simbolizan múltiples beneficios para los usuarios. Sin embargo, su incursión en las escuelas ha sido mucho más lenta de lo que los escolares demandaban. Así, se sostiene que aún tenemos una escuela del siglo XIX o quizás de siglos anteriores, para estudiantes del siglo XXI, que están más conectados que nunca y, en los que se requiere promover habilidades de manejo de información, comunicación, resolución de problemas, pensamiento crítico, creatividad, innovación, autonomía, trabajo en equipo, entre otras (UNESCO, 2015). Por ende, las escuelas se enfrentan a la urgente necesidad de innovar sus modelos educativos y esto, entre otras demandas, requiere de docentes cuya formación inicial y en servicio considere las características de la sociedad actual, lo que redefinirá su papel en el proceso de enseñanza - aprendizaje $y$ en el que las TIC son un aspecto primordial. Por otra parte, y de acuerdo con un análisis llevado a cabo en el 2015 por la Organización para la Cooperación y el Desarrollo Económicos
(OCDE), denominado Estudiantes, Ordenadores y Aprendizaje: Realizando la Conexión, refieren que la exposición de los estudiantes al uso de las computadoras en la escuela no parece ser un factor fundamental para explicar la variación en el desempeño de dichos estudiantes (OCDE, 2015). En esa misma línea, se han realizado numerosos estudios que caracterizan la relación entre TIC y aprendizaje, los mismos que concluyen que más allá de la tecnología está la forma de implementarla como parte de los modelos de enseñanza, pues esta es un medio y no un fin en sí mismo. A la incertidumbre propia de la implementación de las TIC en las escuelas, ahora se suma la asociada a la poca predecibilidad del entorno lo que marca una necesidad de cambio de la concepción de escuela desde un espacio físico - presencial hacia otra post pandemia, de distanciamiento social, donde la tecnología se constituye como nuestro principal aliado. Si bien es cierto, la flexibilidad es una de las características de las TIC, dado que estas permiten atender a tiempos, espacios, ritmos $\mathrm{y}$ formas diferentes de aprender, es necesario preguntarse ¿cuáles son los retos de una escuela en plena transición accidentada hacia la virtualidad mediada por las TIC para estudiantes con NEE, toda vez que esta población demanda de adaptaciones a las estrategias didácticas sustentadas en 
el uso de material concreto, la interacción física entre los docentes y los estudiantes y, el establecimiento de relaciones de cooperación entre el estudiante con NEE, las familias, la comunidad y la sociedad. En ese sentido, este estudio tiene por objetivo identificar información relevante entorno a la experiencia de las madres, padres de familia o tutores sobre el proceso educativo en la modalidad no presencial en el que participan sus hijos, hijas o tutoriados con NEE, así como explorar los retos que demanda la adecuación de la educación en esta modalidad para estudiantes con NEE.

\section{Los estudiantes con Necesidades Educativas Especiales en el Sistema Educativo Peruano}

Nuestro país ha enfrentado cambios importantes en lo que respecta a políticas y lineamientos educativos. Así, la Ley general de educación $\mathrm{N}^{\circ}$ 28044, en su artículo $39^{\circ}$ establece que:

La Educación Básica Especial tiene un enfoque inclusivo y atiende a personas con necesidades educativas especiales, con el fin de conseguir su integración en la vida comunitaria y su participación en la sociedad. Se dirige a (...) personas que tienen un tipo de discapacidad que dificulte un aprendizaje regular. Se imparte con miras a su inclusión en aulas regulares, sin perjuicio de la atención complementaria y personalizada que requieran. (p.12)

En ese sentido, los estudiantes con NEE son aquellos que tienen algún tipo de discapacidad que dificulte un aprendizaje regular. Utilizando el término NEE en su concepción más amplia, este también abarca a estudiantes que presentan Trastorno del Déficit de Atención con Hiperactividad (TDAH), Trastorno del Lenguaje (TL), entre otros diagnósticos planteados desde el punto de vista médico.

En general, el universo de la población tipificada como estudiantes con NEE tiene como posibilidad asistir a las siguientes opciones educativas dentro del sistema educativo peruano como se muestra en la Figura 1.
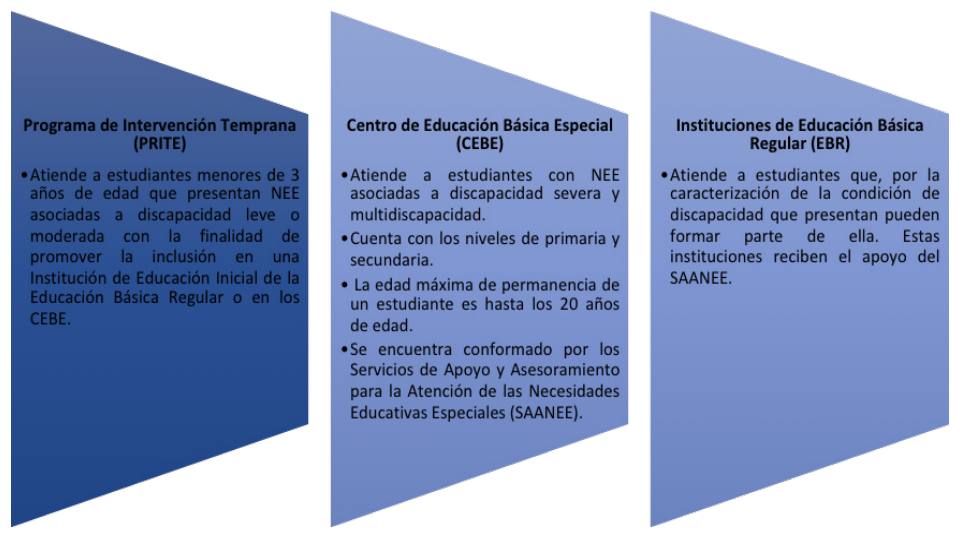

Figura 1. Opciones educativas para estudiantes con NEE en el sistema educativo peruano Fuente: Elaboración propia (2021)

A nivel nacional, según el Ministerio de Educación del Perú (2020) la población con NEE está conformada por 3933 estudiantes que asisten a un PRITE, los cuales son, en su totalidad, de gestión pública. 21247 estudiantes que asisten a un CEBE tanto de gestión pública como privada y 55178 estudiantes en condición de discapacidad se encuentran incluidos en Instituciones de EBR tanto de gestión pública como privada en sus tres niveles: inicial, primaria y secundaria.

Por otro lado, en el marco de la Ley General de la Persona con Discapacidad, Art. 2, los estudiantes con NEE, específicamente aquellos que se encuentran en condición de discapacidad, son personas que tienen una o más deficiencias físicas, sensoriales, mentales o intelectuales de carácter permanente. La discapacidad no es inherente a las personas, sino que, esta discapacidad surge en la interacción entre la persona y el entorno pues es este último quien, al plantear diversas barreras actitudinales y del entorno mismo, origina que las personas en condición de discapacidad no ejerzan o pueden verse impedidas en el ejercicio de sus derechos y su inclusión plena y efectiva en la sociedad, en igualdad de condiciones que las demás personas.

Aunque es necesario centrarse en la persona y no en la diferencia o la condición de discapacidad, es importante tener claridad respecto de la tipificación planteada en los documentos del Ministerio de Educación (MINEDU), para caracterizar a dichos estudiantes.

Según MINEDU (2020a), los estudiantes con NEE pueden presentar las siguientes condiciones 
de discapacidad, como se muestra en la Tabla 1.

Tabla 1. Caracterizaciones de condiciones de discapacidad de los estudiantes con NEE según MINEDU

Necesidades Educativas Especiales asociadas a
Discapacidad

Asimismo, como se observa en la Tabla 2, de las condiciones de discapacidad que plantea el MINEDU, aquella condición presentada por la mayor cantidad de estudiantes inscritos en el sistema educativo peruano para el 2020, es la discapacidad intelectual, en todas las opciones educativas.

Tabla 2. Cantidad de estudiantes por modalidad de estudios y algunas caracterizaciones según MINEDU

\begin{tabular}{lccc}
\hline Caracterización según MINEDU & EBR & $\begin{array}{c}\text { CEBE } \\
\text { (primaria) }\end{array}$ & PRITE \\
\hline Autismo & 4863 & 2041 & 287 \\
Discapacidad auditiva** & 2735 & 227 & 63 \\
Discapacidad intelectual & 30560 & 5499 & 989 \\
Discapacidad motora & 5975 & $*$ & 632 \\
Discapacidad Visual*** & 4903 & 292 & 38 \\
Sordoceguera & 86 & 17 & 3 \\
$\begin{array}{l}\text { Otro tipo u otras NEE asociadas } \\
\text { a discapacidad }\end{array}$ & 6056 & 1637 & 351 \\
Total & $\mathbf{5 5 ~ 1 7 8}$ & $\mathbf{9 7 1 3}$ & $\mathbf{2 ~ 3 6 3}$ \\
\hline
\end{tabular}

* No se registran datos

** Los datos agrupan discapacidad auditiva: hipoacusia y sordera total

*** Los datos agrupan discapacidad visual: baja visión y ceguera

Fuente: elaboración propia, adaptada de MINEDU (2020a)

El proceso educativo de los estudiantes con NEE en el Perú

En el Currículo Nacional de la Educación Básica (CNEB) se pone de manifiesto que, los estándares de aprendizaje declarados para cada una de las competencias que forman parte del perfil de egreso del estudiante, son los mismos para aquellos estudiantes con NEE asociadas a alguna condición de discapacidad. Sin embargo, este documento también visibiliza la necesidad de que el equipo de la Institución Educativa (I.E) empren- da diversas adecuaciones, las cuales pueden ser tanto curriculares como de acceso y/o pedagógicas con el propósito de responder a las características y necesidades especiales particulares de cada uno de los estudiantes en dicha condición, sin que esto involucre bajas expectativas acerca de lo que los estudiantes con NEE pueden lograr. Dada la naturaleza de la población de estudiantes con NEE, se requiere que el trabajo pedagógico se sustente sólidamente en el apoyo de las familias y, las estrategias didácticas, deben partir de los propósitos de aprendizaje en función de las necesidades y características de cada uno de los estudiantes. Existen estrategias para abordar aquellos aspectos a promover en todos los estudiantes, independientemente de la condición de discapacidad que estos presenten. De acuerdo con Minedu (2010), se plantea la siguiente secuencia didáctica que se observa en la Figura 2, la cual es común a todos los estudiantes con NEE asociadas a alguna condición de discapacidad.
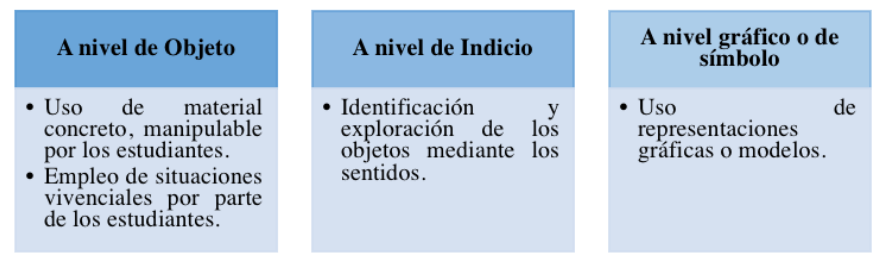

Figura 2. Secuencia para estimular los aprendizajes en diferentes niveles de presentación Fuente: elaboración propia, adaptado de Minedu (2010)

Es importante destacar el rol que cumplen las estrategias didácticas que involucran el uso de materiales concretos y las actividades vivenciales en estudiantes con NEE asociadas a alguna condición de discapacidad, mas aún cuando este tipo de materiales se constituye como un elemento fundamental para la promoción de aprendizajes en esta población estudiantil. Al respecto, MINEDU (2020b), plantea algunas estrategias didácticas pertinentes para estudiantes en determinadas condiciones de discapacidad, todas estas estrategias se encuentran sustentadas en la importancia de la diversidad de canales sensoriales a estimular mediante varios tipos de materiales educativos y, en el caso de la población con NEE, el material concreto es de uso privilegiado y muy frecuente. Así, para promover aprendiza- 
jes acerca de la orientación y el desplazamiento en el espacio, el estudiante en condición de discapacidad visual requiere identificar la información que ingresa por canales sensoriales como el oído (sonidos), el tacto (texturas, relieve) y el olfato (olores) para desarrollar su autonomía. Asimismo, para promover aprendizajes acerca de la necesidad de asociar la palabra con el objeto, estrategias didácticas como la resonancia a través de la manipulación y la identificación de objetos para la asociación tactil - visual son indispensables en estudiantes con NEE. Por lo anterior, cabe reflexionar respecto de cómo impacta la educación virtual en estudiantes en los cuales claramente la experiencia con materiales concretos, el aula y los pares resulta ser indispensable y esencialmente beneficiosa en la promoción de aprendizajes. No se esta exponiendo una dicotomía entre optar por los materiales virtuales o por materiales concretos. Si no más bien, que es fundamental proporcionar al estudiante diversos materiales y no reemplazar unos con otros.

Desde el apogeo de la implementación de la tecnología en el proceso educativo se han ensayado distintos modelos. Sin embargo, es fundamental entender que el que la escuela cuente con tecnología no es un fin en sí mismo, el propósito de esta es ser un medio para la construcción de aprendizajes tanto de los estudiantes como de los docentes y esto demanda de un cambio educativo profundo. Es importante tener claridad respecto de la definición de tecnologías en el marco de la educación de estudiantes con NEE. Según el Servicio Nacional de la Discapacidad (2017) de Chile, una tecnología para inclusión educativa de las personas en situación de discapacidad es:

Cualquier dispositivo, software, equipo, sistema o instrumento fabricado, desarrollado o adaptado que permitan superar y/o eliminar las barreras arquitectónicas, actitudinales y procedimentales que enfrentan las personas en situación de discapacidad durante su desempeño cotidiano, favoreciendo de esta manera su participación e inclusión social a través del ejercicio de derechos humanos. (p.12)
Así, se entiende las TIC en el proceso educativo de estudiantes con NEE, como aquellos dispositivos, software, equipos, sistemas o instrumentos fabricados, desarrollados o adaptados, que facilitan la promoción de aprendizajes en esta población estudiantil. Sin duda alguna, las estrategias didácticas que emprendan los docentes deben sustentarse en la diversidad de características que presentan los estudiantes, especialmente aquellos con NEE y, a su vez, aprovechar los beneficios que brindan las tecnologías para inclusión educativa de las personas en situación de discapacidad. La variabilidad y flexibilidad son aspectos clave de estas, por ello simbolizan un gran potencial para integrarlas, en particular, en el proceso educativo de los estudiantes con NEE. En ese sentido, incorporar en las estrategias didácticas, tanto materiales físicos como virtuales que demanden de tecnología, permitirá abordar esa diversidad de características de la población estudiantil.

Actualmente, se plantea emprender procesos de planificación didáctica de acuerdo con el Diseño Universal para el Aprendizaje (DUA), el cual propone promover aprendizajes en los estudiantes ofreciendo los contenidos en múltiples medios, así como apoyos integrados e instantáneos: glosarios accesibles por hipervínculos, información previa y asesoramiento en pantalla, es decir, el uso de las TIC (Minedu, 2020b). El DUA permite no solo atender de manera diferenciada a los estudiantes con NEE asociadas a alguna condición de discapacidad, sino que, también permite potenciar las estrategias didácticas para la promoción de aprendizajes en estudiantes que no presentan dichas condiciones.

En el contexto actual, las TIC han pasado a tener un papel central en el proceso educativo. En ese sentido, existe multiplicidad de materiales virtuales que facilitan el proceso de enseñanza aprendizaje. De acuerdo con UNESCO (2011), "el uso de TIC accesibles se destaca como un instrumento clave para ofrecer a los estudiantes medios de enseñanza acordes con sus aptitudes y estilos de aprendizaje individuales" (p.5). La incorporación de las TIC en el proceso de enseñan$\mathrm{za}$ - aprendizaje permite reducir las barreras de la 
discapacidad y genera oportunidades para desarrollar la autonomía de los estudiantes con NEE asociadas a alguna condición de discapacidad toda vez que brinda herramientas para atender de manera diferenciada a las necesidades y características particulares. Sin embargo, el gran reto en relación con la incorporación de las TIC en el proceso educativo de estos estudiantes, involucra aspectos económicos para la accesibilidad a este tipo de tecnologías, además de la formación de los profesionales que, de manera interdisciplinaria, participan del proceso educativo.

En sentido amplio, además de las tecnologías convencionales para estudiantes con NEE, como es el caso del bastón para que estudiantes con discapcidad visual se desplacen con autonomía, los parlantes, las lupas o magnificadores portátiles, el punzón y regleta, entre otros, en la actualidad, los sistemas operativos ya incluyen herramientas que permiten facilitar el acceso a la información por parte de estudiantes con NEE, como por ejemplo, algunas combinaciones de teclas que permiten magnificar la pantalla para una mejor visualización, narrar la información de la pantalla de la PC o, redactar la información que el usuario oraliza. Adicional a lo anterior, diversos programas que facilitan el acceso a la información por parte de los estudiantes con NEE, se encuentran disponibles, ya sea en línea o de descarga (libre o con costo). A continuación, en la Tabla 3 se presentan algunas TIC, de acceso libre, para la promoción de aprendizajes en esta población.

Tabla 3. Algunas TIC para la promocion de aprendizajes en los estudiantes NEE

\begin{tabular}{|c|c|c|}
\hline $\begin{array}{l}\text { Condición de } \\
\text { discapacidad }\end{array}$ & $\begin{array}{c}\text { TIC en el proceso } \\
\text { educativo de } \\
\text { estudiantes con NEE }\end{array}$ & Webs \\
\hline $\begin{array}{l}\text { Discapacidad } \\
\text { visual }\end{array}$ & $\begin{array}{l}\text { Programas de ampliación } \\
\text { de texto en pantalla de } \\
\text { ordenador }\end{array}$ & $\begin{array}{l}\text { http://texttospeechrobot.com/tts/es/texto-a-voz/ } \\
\text { https://www.texttomp3.online/index-es.html }\end{array}$ \\
\hline $\begin{array}{l}\text { Discapacidad } \\
\text { auditiva }\end{array}$ & $\begin{array}{l}\text { Conversores de texto a } \\
\text { voz } \\
\text { https://www.dictation.io/s } \\
\text { peech }\end{array}$ & https://www.dictation.io/speech \\
\hline $\begin{array}{c}\text { Trastorno del } \\
\text { Especto Autista }\end{array}$ & $\begin{array}{l}\text { Aplicaciones basadas en } \\
\text { sistemas de comunicación } \\
\text { aumentativa o alternativa } \\
\text { o pictogramas }\end{array}$ & $\begin{array}{l}\text { https://www.pictotraductor.com } \\
\text { http://aulaabierta.arasaac.org }\end{array}$ \\
\hline
\end{tabular}

Fuente: elaboración propia (2021)
La educación virtual y las familias de los estudiantes con NEE

La educación virtual es un proceso en el que se pretende poner en juego diversas acciones pedagógicas para generar aprendizajes en los estudiantes. Tanto las acciones pedagógicas como las acciones que los estudiantes realizan, se encuentran mediadas por las TIC. De acuerdo con Juca (2016), esta modalidad se caracteriza por ser flexible, dinámica y adaptativa al medio donde se desarrolla. Asimismo, necesita y procura un estudiante "responsable y creativo en la construcción de su aprendizaje, que adopte estrategias y estilos de aprendizaje a partir del conocimiento de sí mismo, a medida que lo logre aprenderá a aprender" (Juca, 2016, p.108).

Por otra parte, de acuerdo con Oñate, Reyes y Villarroel (2016), la participación de los padres en la escuela, antes de la pandemia por la COVID 19, había sido desde perspectivas como: i. una visión de participación entendida como comunicación con la escuela y apoyo de los aprendizajes del aula, en la realización de las tareas escolares; ii. una perspectiva que añade a la visión anterior la dimensión de provisión de recursos educativos, en tanto que esto involucra proporcionarles a sus hijos actividades fuera de la escuela, pero orientadas al enriquecimiento de sus aprendizajes. Sin embargo, la educación virtual a causa de la pandemia ha dinamizado completamente estas perspectivas antes existentes.

Por su parte, la educación a distancia destaca por su flexibilidad en diversos sentidos, lo cual resulta ser un beneficio para el usuario que la recibe. Sin embargo, elementos como sensaciones, anhelos y emociones que se transmiten en la educación presencial son muy difíciles de manifestar e identificar a distancia (Martínez, 2017). Dicha flexibilidad demanda de los estudiantes, no solo diversos procesos cognitivos sino también de funciones metacognitivas superiores, como la autorregulación; es decir, la capacidad para gestionar el nivel de la propia energía de acuerdo con las exigencias de una tarea o situación (Shan, 2013), y en ello las familias juegan un rol impres- 
cindible toda vez que, ahora se trata de estudiantes cuyo proceso educativo se ve mediado por la tecnología sin el acompañamiento físico del docente.

Para responder al contexto actual, desde las escuelas de gestión pública el Ministerio de Educación ha planteado y puesto en marcha diversas normas y estrategias, entre las cuales se destaca la estrategia nacional y, a su vez, plataforma Aprendo en Casa para las distintas modalidades de la EBR, entre ellas, aquellas opciones en las que se atiende a estudiantes con NEE.

En las orientaciones brindadas por el MINEDU (2020b), se explicita que, para el desarrollo de estas actividades en casa, se requiere del apoyo de un adulto, es decir, el rol de la madre, padre o tutor y, el uso de materiales concretos y situaciones vivenciales. Así, el papel de la familia es aun más esencial y más activo que en la educación presencial y, sobre todo, tratándose de estudiantes con NEE. En ese sentido, la madre, padre o tutor del estudiante deben desempeñarse como apoyo primordial en la implementación de esta estrategia nacional. La programación de actividades para estudiantes con NEE aborda básicamente las siguientes áreas, como se muestra en la Figura 3.

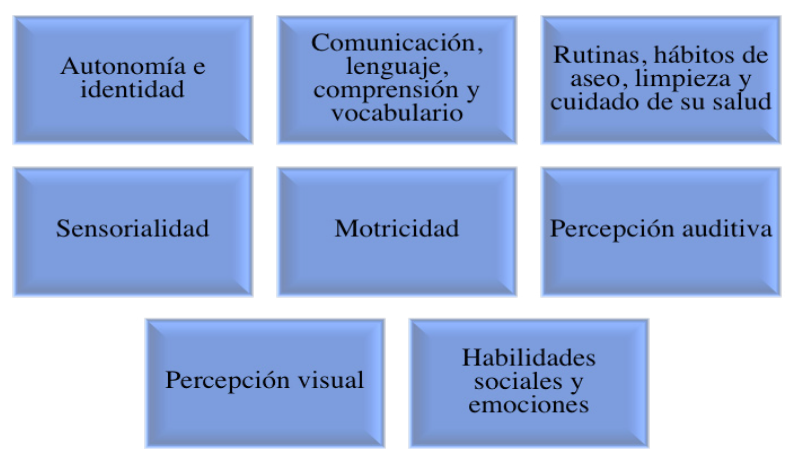

Figura 3. Áreas a promover en los estudiantes con NEE a través de la estrategia Aprendo en Casa Fuente: elaboración propia (2021)

Sin duda, el hogar es la primera escuela de las personas. Los patrones de crianza en términos de las oportunidades de aprendizaje que significan para los niños y las niñas influyen en su proceso educativo formal, en la escuela. A su vez, la familia es un soporte determinante durante el proceso educativo en la escuela presencial pues, producto de esta relación los estudiantes obtienen resulta- dos educativos positivos (Razeto, 2016).

La influencia de la familia en el proceso educativo se evidencia en diversas áreas del desarrollo de los niños y las niñas como en aquellos citados por López y Guaimaro (2015); el comportamiento social en la escuela, el logro de aprendizajes, la motivación para aprender, entre otros. Adicional a lo anterior, Pinto (2016) destaca a la famila como aquel espacio donde tiene lugar el inicio en la formación de valores en los niños y las niñas a través del sistema de costumbres, tradiciones y reglas que se encuentran en ella. Estas perspectivas son muy interesantes pues estaban descritas en función de la escuela presencial de tal manera que la responsabilidad de los aprendizajes estaba centrada en los docentes y, en el hogar, las madres, padres o apoderados contribuían en la medida de sus posibilidades al proceso de educación siendo un soporte para la escuela. Sin embargo, el contexto actual traslada en mayor medida la responsabilidad del proceso educativo al hogar. Así, esta situación demanda que la escuela, esta vez, a través de la tecnología se inserte en la dinámica familiar y ello amerita un trabajo coordinado para poder garantizar el éxito en los aprendizajes (Martorell y Aliagas, 2020).

Respecto al papel de las familias en el proceso educativo de los estudiantes con NEE es fundamental, sobre todo, para aquellos que tienen asociado alguna condición de discapacidad. La familia como un sistema (Bronfenbrenner como se citó en Shelton, 2018), soporta las presiones, tanto del ambiente externo como interno, que afectan directamente en la dinámica familiar $y$, en los otros sistemas. En ese sentido, estudios previos señalan que las madres de los estudiantes con NEE experimentan tensiones psicológicas, las cuales se incrementan o disminuyen en relación directa con la perccepción del progreso de su hijo o hija con NEE (Shea y Bauer, 2000).

El proceso educativo de un estudiante con NEE demanda del establecimiento de un sistema de colaboración entre la escuela y la familia dado que se requiere de una preparación especializada en el abordaje de las necesidades, no solo educativas, de estos estudiantes. Sin embargo, es importante mencionar que la disposición de los 
padres de familia y la accesibilidad a programas educativos orientados a estudiantes con NEE se ve fuertemente vinculado con aspectos socioeconómicos.

\section{Materiales y método}

\section{Participantes}

Se seleccionó una muestra no probabilística. Se eligieron aquellas madres, padres o tutores de estudiantes con NEE que, luego de haber visualizado la invitación a participar de la investigación en medios virtuales como comunidades virtuales de padres de familia con niños con NEE, comunidades virtuales de docentes de estudiantes con NEE, instituciones con y sin fines de lucro con servicios orientados a la atención de estudiantes con NEE, optaron por completar el cuestionario. Así, la muestra quedó conformada por $30 \mathrm{ma}-$ dres, padres o tutores de estudiantes con NEE cuyas edades se encontraban entre 6 a 12 años.

\section{Instrumento}

Entre las características fundamentales a explorar acerca de los retos de una educación virtual para estudiantes con NEE en las preguntas del cuestionario, se encuentran:

- Uso de la tecnología por parte del estudiante con NEE: manejo de funcionalidades de la tecnología, interacción con objetos virtuales, gustos y preferencias respecto de alguna de estas funcionalidades, así como las dificultades asociadas a la misma.

- Establecimiento de relaciones interpersonales: docente - estudiante con NEE, estudiante con NEE - estudiantes integrantes del aula virtual de clases. Logros y dificultades en el establecimiento de dichas relaciones.

- Procesos cognitivos asociados al aprendizaje mediante el uso de tecnologías: Atención, concentración, aprendizajes alcanzados. Logros y dificultades respecto de los procesos cognitivos en el campo educativo.

- Retos y aspectos de mejora del uso de tecnologías para el proceso educativo de los estudiantes con NEE.
Asimismo, se vio conveniente incluir dos preguntas que exploraban las percepciones de las madres, padres o tutores de estudiantes con NEE acerca de la educación virtual de la que participaron durante el 2021. Además, el cuestionario recoge información sobre el género, la edad, país de escolaridad durante el 2020, la NEE de la estudiante asociada a alguna condición de discapacidad. La validez del instrumento fue realizada por 2 expertos en el tema y la confiabilidad se realizó mediante el Alpha de Cronbach.

\section{Tipo de estudio}

El presente estudio es de diseño no experimental, transeccional y de nivel exploratorio, al respecto Hernández, Fernández y Baptista (2014) señalan que los estudios exploratorios se realizan cuando los eventos y fenómenos a investigar son recientes o nuevos.

\section{Procedimiento}

Se inició con la identificación del tema a investigar teniendo en cuenta las condiciones cambiantes que surgieron en la educación en NEE. Luego, se realizó entrevistas y cuestionarios a algunas madres, padres o tutores de estudiantes con NEE que habían tenido una educación virtual durante todo el 2020, a consecuencia de la pandemia por la COVID - 19. Fue a partir de estas entrevistas y cuestionarios, que se determinó que información se deseaba recopilar con el estudio y y elaborar el cuestionario situado en la investigación e iniciar el proceso de validación y confiabilidad. En el mes de enero 2021, teniendo claro los objetivos del estudio se aplicó los instrumentos en febrero, a través de medios como redes sociales y aplicaciones de mensajería instantánea.

\section{Consentimiento informado}

Al momento de compartir dicho cuestionario, los madres, padres o tutores de estudiantes con NEE recibieron un mensaje con una explicación breve acerca del estudio. El registro de información en el cuestionario fue anónimo, aunque, si 
es que el participante lo deseaba, podría también colocar su correo electrónico. Los participantes fueron adultos y, estuvieron informados de los fines que presentó dicho instrumento de investigación, a través de una breve y clara descripción cuando se les envío el enlace.

\section{Resultados}

Dado que el objetivo del presente estudio de carácter exploratorio consiste en identificar información relevante respecto de la experiencia de las madres, padres de familia o tutores acerca del proceso educativo en la modalidad no presencial, mediado por la tecnología, del que participan sus hijos, hijas o tutorados con NEE así como los retos que esta modalidad plantea, se presentan los resultados teniendo en cuenta información sociodemográfica y aspectos fundamentales referidos por los padres, madres o tutores, en relación con los retos que presenta la educación virtual en la que participaron los niños (as) con NEE.

Tabla 4. Edades de los niños(as) con NEE cuyos padres participaron en la investigación

\begin{tabular}{ccc}
\hline Descriptivos & Media & D.E. \\
\hline & 9,70 & 2,261 \\
\hline Frecuencias & $\mathrm{f}$ & $\%$ \\
\hline 6 - 7 años & 8 & 26,6 \\
$8-9$ años & 4 & 13,4 \\
$10-12$ años & 18 & 60,0 \\
\hline Total & 30 & 100,0 \\
\hline
\end{tabular}

Fuente: elaboración propia (2021)

Como se observa en la Tabla 4, la muestra de la presente investigación estuvo conformada por madres, padres o tutores de estudiantes con NEE cuyo rango de edad se encontraba entre los 6 y 12 años $y$, en edad de recibir educación virtual durante el 2020.

En relación con el proceso de educación virtual de los niños(as) con NEE cuyas madres, padres o tutores participaron del presente estudio, se evidenció que, aunque estaban inscritos y habían iniciado el proceso de escolarización 2020 en la modalidad de educación virtual en marzo (por $\mathrm{TV}$, por teléfono, a través de mensajería instan- tánea, plataforma virtual o cualquier otra tecnología), en algunos casos decidieron retirarlos, como se observa en la Tabla 5. Al explorar cuáles fueron las razones por las que las madres, padres o tutores retiraron a los niños(as) con NEE de la educación virtual, se pudo identificar que el motivo principal de esa decisión fue la percepción de que sus hijos(as) no estaban aprendiendo y, por lo tanto, no era necesario invertir recursos en el proceso educativo escolarizado y virtualizado durante el 2020.

Tabla 5. Participación del niño(a) con NEE en la educación vir-

\begin{tabular}{ccc}
\hline $\begin{array}{c}\text { Participación del } \\
\text { estudiante en la educación } \\
\text { virtual }\end{array}$ & f & $\%$ \\
\hline No & 5 & 16,7 \\
Sí & 25 & 83,3 \\
\hline Total & 30 & 100,0 \\
\hline Fuente: Elaboración propia (2021)
\end{tabular}

Entre las condiciones de discapacidad tipificadas según el Ministerio de Educación y, agrupadas para efectos de esta investigación como se muestra en la Tabla 6, se evidenció con mayor frecuencia en la muestra, el Trastorno del Espectro Autista (Autismo, en cualquier grado) y la Discapacidad Intelectual (asociada o no al Síndrome de Down u otro síndrome). Estas condiciones de discapacidad fueron referidas por las madres, padres o tutores de los estudiantes con NEE. El 89,7\% de los estudiantes con NEE había sido diagnosticado por un profesional de la salud (psicóloga, neurólogo, neuropediatra, psiquiatra). Las madres, padres o tutores del $10,3 \%$ de los estudiantes con NEE consignó un diagnóstico sustentado en diversos criterios personales más no estaba referido a un diagnóstico realizado por un profesional de la salud.

Tabla 6. Condición del niño(a) con NEE

\begin{tabular}{ccc}
\hline Condición de discapacidad del estudiante & f & \% \\
\hline Trastorno del Déficit de Atención sin Hiperactividad & 1 & 3,3 \\
Discapacidad Intelectual (con o sin Síndrome de Down u otro & 8 & 26,7 \\
$\quad$ síndrome) & & \\
Trastorno del Espectro Autista (Autismo, en cualquier grado) & 13 & 43,3 \\
Multidiscapacidad (Dos o más opciones de esta lista) & 4 & 13,3 \\
Problemas del lenguaje & 1 & 3,3 \\
Trastorno del déficit de atención con hiperactividad & 3 & 10,0 \\
\hline Total & 30 & 100,0 \\
\hline Fuente: Elaboración propia (2021) & &
\end{tabular}


Respecto de los aspectos fundamentales explorados acerca de los retos de la educación virtual para estudiantes con NEE durante el 2020, se obtuvieron los resultados que se presentan en la Tabla 7.

Tabla 7. Percepción del proceso de adaptación del niño(a) con NEE a la educación virtual

\begin{tabular}{ccc}
\hline $\begin{array}{c}\text { Adaptación del estudiante con } \\
\text { NEE a la educación virtual }\end{array}$ & $\mathbf{f}$ & $\mathbf{\%}$ \\
\hline Se adaptó muy bien & 1 & 3,3 \\
Se adaptó bien & 4 & 13,3 \\
Su adaptación fue regular & 9 & 30,0 \\
No se adaptó & 11 & 36,7 \\
Lo tuve que retirar & 5 & 16,7 \\
\hline Total & 30 & 100,0 \\
\hline Fuente: Elaboración propia (2021) & \\
\hline
\end{tabular}

Como parte de la información relevante como recogida en el presente estudio, se identificó que el 36,7\% de las madres, padres o tutores de niños con NEE decidieron que estos continúen el proceso de educación virtual, a pesar de que no se pudieron adaptar a la nueva modalidad. Un importante grupo de estudiantes también continuó con dicho proceso de escolarización a pesar de presentar una regular adaptación al mismo.

Tabla 8. Dificultades evidenciadas en el niño(a) con NEE durante la educación virtual

\begin{tabular}{ccc}
\hline $\begin{array}{c}\text { Dificultad durante } \\
\text { las clases virtuales }\end{array}$ & F & $\%$ \\
\hline $\begin{array}{c}\text { Ninguna } \\
\text { Falta de atención y } \\
\text { concentración }\end{array}$ & 21 & 10,0 \\
Falta de motivación & 6 & 20,0 \\
\hline Total & 30 & 100,0 \\
\hline Fuente: Elaboración propia (2021)
\end{tabular}

Como se evidencia en la Tabla 8 , el $70 \%$ de estudiantes con NEE manifestaron como principal dificultad la falta de atención y concentración durante el proceso de educación virtual en el 2020. Como parte de la exploración del presente estudio respecto del papel de la madre, padre o tutor de estos estudiantes durante el proceso de educación virtual, el 100\% de ellos destacaron que el niño(a) dependía totalmente de la presencia de un adulto durante todas las actividades desarrolladas en dicho proceso, aun así, ellos manifestaban falta de atención, concentración, escasa disposición y motivación para involucrarse en las tareas que demandaba la educación virtual por parte de los estudiantes con NEE.

Tabla 9. Percepción sobre el logro de aprendizajes no relacionados al uso de las TIC del niño(a) con NEE mediante la educación virtual

\begin{tabular}{ccc}
\hline $\begin{array}{c}\text { Logros de aprendizaje de los(as) } \\
\text { estudiantes con NEE }\end{array}$ & F & $\%$ \\
\hline No & 18 & 60,0 \\
Sí & 12 & 40,0 \\
\hline Total & 30 & 100,0 \\
\hline Fuente: elaboración propia (2021)
\end{tabular}

Respecto de los aprendizajes logrados por los estudiantes con NEE, como se evidencia en la Tabla 9 , el 60\% de los participantes refirieron que, en su mayoría estos aprendizajes estaban relacionados con el uso de las herramientas tecnológicas. Sin embargo, no fue necesario que los docentes medien en dicho aprendizaje pues los niños(as) con NEE lo lograron a través de uso que le daban.

Entre las actividades didácticas asociadas a las TIC preferidas por el niño(a) con NEE se destaca que los juegos interactivos, los videos de corta duración (menos de 3 minutos aproximadamente), audio libros, son aquellas actividades de mayor agrado para esta población estudiantil. Sin embargo, las madres, padres o tutores de estos estudiantes destacaron el rol fundamental que cumplen los materiales concretos y la orientación de cómo usarlos en casa, por parte una docente con experticia en niños con NEE. El grupo de participantes que refirió haber retirado a sus hijos(as) del proceso de escolarización 2020 de la educación virtual señaló que sus niños(as) logran mayores aprendizajes en el día a día en el hogar, que cuando media el uso de la tecnología.

Tabla 10. Relación entre el(la) docente y el(la) niño(a) con NEE durante la educación virtual

\begin{tabular}{|c|c|c|}
\hline Interacción docente - niño(a) con NEE & f & $\%$ \\
\hline Interacción frecuente docente - estudiante con NEE & 10 & 33,3 \\
\hline $\begin{array}{l}\text { Interacción regular o poca interacción docente - estudiante } \\
\text { con NEE }\end{array}$ & 8 & 26,7 \\
\hline Nula interacción docente - estudiante con NEE & 11 & 36,7 \\
\hline No especifica & 1 & 3,3 \\
\hline Total & 30 & 100,0 \\
\hline
\end{tabular}

Fuente: Elaboración propia (2021) 
Como se presenta en la Tabla 10, respecto de la relación entre el (la) docente y el (la) estudiante con NEE durante la educación virtual como uno de los hallazgos a partir del análisis de las respuestas abiertas del cuestionario, se evidencia que, los participantes atribuyen una relación entre las aulas donde la población estudiantil con el establecimiento de una interrelación más frecuente entre la docente y el(la) niño(a) con NEE. Sin embargo, el 63,4\% de los participantes de la presente investigación señala que esta interrelación docente - estudiante durante la educación virtual ha sido poco frecuente o nula.

Tabla 11. Relación entre el(la) niño(a) con NEE y los estudiantes durante la educación virtual

\begin{tabular}{lcc}
\hline $\begin{array}{l}\text { Interacción niño(a) con NEE - otros } \\
\text { niños }\end{array}$ & f & $\%$ \\
\hline $\begin{array}{l}\text { Interacción frecuente entre estudiante con } \\
\text { NEE - otros estudiantes }\end{array}$ & 5 & 16,7 \\
$\begin{array}{l}\text { Interacción regular o poca interacción } \\
\text { entre estudiante con NEE - otros }\end{array}$ & 4 & 13,3 \\
estudiantes & & \\
$\begin{array}{l}\text { Nula interacción entre estudiante con } \\
\text { NEE - otros estudiantes }\end{array}$ & 21 & 70,0 \\
\hline \multicolumn{1}{c}{ Total } & 30 & 100,0 \\
\hline
\end{tabular}

Fuente: Elaboración propia (2021)

Una de las características fundamentales de la escuela antes de la pandemia por la COVID 19, era el proporcionar un espacio de socialización y construcción de vínculos con los pares. Durante la educación virtual este espacio de socialización que antes proporcionaba la escuela no virtual definitivamente sufrió cambios drásticos, tal es que, como se evidencia en la Tabla 11, el 83,3\% de los participantes señala que la interacción entre los estudiantes fue poca o nula.

Acerca de los retos de la educación virtual mediante TV, teléfono, mensajería instantánea o alguna plataforma virtual ya sea open o close source, para estudiantes con NEE, se obtuvieron los resultados de la Tabla 12. Llama la atención que uno de los principales retos reportados por los participantes plantee la necesidad de encontrar, de alguna manera, espacios físicos de trabajo pedagógico con los estudiantes con NEE. Este reto es incompatible con la modalidad virtual, pero resulta ser tan esencial para esta población que se consigna como respuesta del cuestionario. Esto último abre la posibilidad de una modalidad semi presencial.

Tabla 12. Retos de la Educación virtual para estudiantes con NEE

\begin{tabular}{ccc}
\hline Retos para la educación especial virtual & f & $\%$ \\
\hline Generar espacios de contacto físico entre el/la docente y el(la) estudiante con NEE & 12 & 40,0 \\
\hline $\begin{array}{c}\text { Generar espacios de socialización entre el(la) estudiante con NEE y los compañeros } \\
\text { de clase }\end{array}$ & 2 & 6,67 \\
\hline Generar aprendizajes en el(la) estudiante con NEE, a través de la educación virtual & 3 & 10,00 \\
\hline Desarrollar estrategias didácticas usando las TIC para el(la) estudiante con NEE & 4 & 13,33 \\
\hline $\begin{array}{c}\text { Desarrollar estrategias didácticas usando las TIC para el(la) estudiante con NEE, en } \\
\text { zona rural }\end{array}$ & 3 & 10,00 \\
\hline Desarrollar estrategias didácticas como docentes y padres de familia usando las & 6 & 20,00 \\
\hline TIC para el(la) estudiante con NEE & 30 & 100,0 \\
\hline Total & & \\
\hline
\end{tabular}

Fuente: elaboración propia (2021)

Los participantes manifestaron necesidades asociadas con el trabajo terapéutico con sus hijos(as), así como la necesidad de atención a las características diferentes respecto del resto del aula, sobre todo cuando estos estudiantes se encuentran en la modalidad de incluido en la EBR. Asimismo, los participantes destacaron la necesidad de generar espacios de socialización entre estudiantes, mediados o no por las TIC. Por otra parte, las madres, padres o tutores de niños(as) con NEE enfatizan en la necesidad de formación docente en estrategias didácticas para generar aprendizajes en esta población y, ahora, mediar este proceso educativo a través de las TIC pues, como es evidente, anteponen la salud física y para ello aceptan el distanciamiento social pertinente por la pandemia.

Tabla 13. Necesidades de los padres de niños(as) con NEE para participar de la Educación Virtual

\begin{tabular}{lcc}
\hline \multicolumn{1}{c}{ Necesidades de los PPFF } & f & \% \\
\hline $\begin{array}{l}\text { Apoyo de especialistas en niños(as) con NEE } \\
\text { Asociadas a las estrategias para generar }\end{array}$ & 7 & 23,3 \\
$\begin{array}{l}\text { aprendizajes en el niño(a) con NEE } \\
\text { Asociadas a las herramientas tecnológicas } \\
\text { (disponibilidad y manejo) }\end{array}$ & 13 & 63,3 \\
$\begin{array}{l}\text { Reorganización de las actividades del adulto } \\
\text { que acompaña al niño(a) con NEE }\end{array}$ & 8 & 26,7 \\
\multicolumn{1}{c}{ Total } & 30 & 100,0 \\
\hline
\end{tabular}

De acuerdo con la Tabla 13, el 43\% de los participantes puntualizaron la necesidad de adaptar las propuestas pedagógicas que se difunden a tra- 
vés de las TIC a entornos rurales, o en aquellos en donde, por diversos factores, principalmente económicos, no se dispone de herramientas TIC.

\section{Discusión y conclusiones}

La Educación virtual actual ha surgido por las presiones que demanda el contexto de pandemia en el que estamos viviendo. Definitivamente, se esta aprendiendo a partir de la evidencia y sobre la marcha. Es importante tener en cuenta que estas lecciones aprendidas se deben de evaluar no solo desde su impacto en la Educación Básica Regular sino también, desde la complejidad de la Educación Básica Especial.

La investigación realizada por Solís (2020), analizó la inclusión escolar ante los desafíos del COVID-19, a efectos de intervenir y atender de manera adecuada las necesidades especiales de los niños. Como resultados, se planteó que existe una tendencia desfavorable en lo que se refiere a la aplicación de la educación inclusiva; más aun cuando la pandemia ha obstaculizado la educación de esta población estudiantil. La presente investigación sostiene también que el contexto de educación virtual, más aún durante la pandemia por la COVID -19, ha afectado negativamente el proceso educativo de los estudiantes con NEE.

Por otra parte, Ramírez, Maldonado y Villacorta (2020) utilizaron un cuestionario de preguntas abiertas como instrumento de recolección de datos para una muestra de 36 madres y 2 padres de niños y adolescentes con discapacidad para identificar las principales ventajas y desventajas que advierten en este periodo donde la escuela se está desarrollando desde sus hogares. Entre los resultados, se destacan que los participantes identifican el valor de involucrarse de manera activa en el proceso educativo de sus hijos. Sin embargo, advierten la necesidad de desarrollar procesos de formación respecto de la educación de estudiantes con NEE, sobre todo porque en este proceso existe una desarticulación entre la escuela y las familias. Al respecto, esos hallazgos coinciden con los encontrados en la presente investigación en relación con la necesidad de formación de los miembros de la comunidad educativa para po- der responder de manera pertinente a las necesidades educativas de esta población, sobre todo, cuando de educación virtual se trata. Así mismo, los autores mencionados, destacan la carente interacción de sus hijos con sus pares, la falta de comunicación entre la escuela y ellos, como desventajas de la educación virtual para estudiantes con NEE. Retos con los que también coincidimos como hallazgos de la presente investigación.

El presente estudio pone en evidencia que principalmente la gestión del tiempo, las estrategias y recursos didácticos, la relación de cooperación con las familias de los estudiantes con NEE, las adecuaciones de las políticas y lineamientos educativos, así como la adecuación de las estrategias y recursos didácticos a diversas realidades de nuestro país, forman parte de los grandes retos de la educación virtual para esta población estudiantil. Retos que, por derecho se requiere cubrir. Estos hallazgos coinciden con diversas investigaciones sustentadas en la revisión de la literatura, como la realizada por Crisol, Herrera y Montes (2020), denominada: Educación virtual para todos, en la cual se plantea la necesidad de proponer acciones pedagógicas que contemplen materiales elaborados en función del diseño como el DUA, es decir, orientados a la inclusión educativa. A su vez, esta investigación también destaca el papel fundamental de la familia y la necesidad de su capacitación para su participación activa en el proceso educativo de los estudiantes con NEE, lo que coincide con la investigación bibliográfica planteada por Rodas y Gómez (2020), denominada Discapacidad Intelectual. Reflexiones sobre la inclusión en educación virtual.

En definitiva, la educación virtual en el contexto de la pandemia ha significado cambios drásticos en la dinámica familiar y en el papel que los integrantes del contexto familiar desempeñan en la educación virtual del niño(a) con NEE. Así, las madres, padres o tutores advierten que, dado el compromiso que han asumido con la educación virtual de su niño(a) con NEE, algunos de ellos han tenido que prescindir de sus puestos laborales, lo cual ha tenido un impacto negativo en la economía de sus hogares. Asimismo, los 
participantes del presente estudio demandan el apoyo de especialistas tanto en el trabajo directo con los(las) niños(as) como en la orientación de cómo desarrollar las actividades escolares, tanto las relacionadas con el uso de las TIC como las vinculadas al uso de material concreto.

Es importante que las familias se involucren en el proceso educativo de los estudiantes con NEE, pero para ello, se requiere brindarles las herramientas necesarias, en relación con el conocimiento, recursos y atención a la individualidad de cada niño(a), a fin de responder a las diversas necesidades de estos(as) niños(as) y, lograr aprendizajes en ellos. Estas conclusiones son de carácter relevante y fundamental al momento de plantear e implementar lineamientos y políticas orientadas a la educación de los estudiantes con NEE toda vez que, esta población demanda de una atención diferenciada donde las TIC pasa a segundo plano pues, los servicios terapéuticos o espacios de socialización física no son susceptibles de cubrir usando este tipo de recursos.

\section{Referencias Bibliográficas}

Crisol, E., Herrera, L. y Montes, S. (2020). Educación virtual para todos: una revisión sistemática. Education in the Knowledge Society, 21. https:// doi.org/10.14201/eks.23448

Hernández, S., Fernández, C. y Baptista P. (2014). Metodología de la Investigación. Mc Graw Hill Interamericana. México.

Juca, F. (2016). La educación a distancia, una necesidad para la formación de los profesionales. Revista Universidad y Sociedad [seriada en línea], 8 (1).pp.106-111. http://rus.ucf.edu.cu/ Ley General de Educación No 28044. Diario Oficial de la República de Perú, Lima, Perú, 2 de septiembre de 2009.

Ley General de la Persona con Discapacidad No 29973. Diario Oficial de la República de Perú, Lima, Perú, 7 de abril de 2014.

López, G. y Guaimaro, Y. (2015). El rol de la familia en los procesos de educación y desarrollo humano de los niños y niñas. Ixaya Revista Universitaria de Desarrollo Social. http://revistascientificas.udg.mx/index.php/IXA/article/ view/6742/5822

Martínez, V. (2017). Educación presencial versus educación a distancia. La Cuestión Universitaria, 9. 2017, pp. 108-116.

Martorell, C. y Aliagas, C. (2020). El vaivén de los portátiles entre las aulas y el hogar

la perspectiva de las familias sobre la reforma educativa de la Escuela 2.0. Revista complutense de educación, 31, (1), 127-135. https://doi. org/10.5209/rced.61922

Ministerio de Educación del Perú. (2010). Guía Para Orientar la Intervención de los Servicios de Apoyo y Asesoramiento para la Atención de las Necesidades Educativas Especiales SAANEE. Dirección General de Educación Básica Especial. Ministerio de Educación del Perú (2016). Currículo Nacional de la Educación Básica. Perú.

Ministerio de Educación del Perú (2020a). Oficina de Atención al Ciudadano y Gestión. Unidad de Estadística. Datos solicitados y enviados a través de plataforma de libre acceso a la información pública.

Ministerio de Educación del Perú. (2020b). Curso virtual del uso de materiales para la atención de estudiantes en condición de discapacidad atendidos por los CEBE Y SAANEE a nivel nacional. Módulo 1. Dirección General de Educación Básica Especial.

OCDE. (2015). Students, Computers and Learning. Making the Connection. Paris: OCDE Publishing. https://doi.org/10.1787/9789264239555-en

Oñate, A., Reyes, F. y Villarroel, V. (2016). Participación y expectativas de los padres sobre la educación de sus hijos en una escuela pública. Estudios pedagógicos, 42 (3). https://doi. org/10.4067/S0718-07052016000400019

Pinto, R. (2016). La importancia de promover los valores del hogar hacia las escuelas primarias. Revista Ra Ximhai, 12, (3), 271-283. https://doi. org/10.35197/rx.12.02.2016.18.rp

Ramírez, I., Maldonado, C. y Villacorta, R. (2020). De la Jaula al Aula abierta: Niños con discapacidad. Infancias: Discapacidad e interseccionalidades, 3 (3), 1-7.

Razetto, A. (2016). Estrategias para promover la participación de los padres en la educación 
de sus hijos: el potencial de la visita domiciliaria. Estudios pedagógicos, 42 (2). https://doi. org/10.4067/S0718-07052016000200026

Rodas, D. y Gómez, M. (2020). Discapacidad Intelectual. Reflexiones sobre la inclusión en educación virtual. Ensayos de filosofía, 12 (2).

Servicio Nacional de la Discapacidad de Chile. (2017). Catálogo de Tecnologías para la Educación Inclusiva.

Shanker, S. (2013). Calma, Atención y Aprendizaje. Ontario, Canadá: Pearson Education.

Shea, T. y Bauer, A. (2000). Educación Especial. Un enfoque ecológico. Mc Graw Hill.

Shelton, L. (2018). The Bronfenbrenner Primer. A Guido to develecology. Routledge.

Solís-Mejías J. (2020). La inclusión escolar ante los desafíos del COVID-19. Caso: Colegio Juan Ignacio Molina, Chile. Revista Ethos, 1(1), 86101. http://ojs.udelistmo.edu/ojs/index.php/ Ethos/article/view/114

UNESCO, (2011). Las TIC accesibles y el aprendizaje personalizado para estudiantes con discapacidad: Un diálogo entre los educadores, la industria, el gobierno y la sociedad civil. Informe sobre la Reunión Consultiva de Expertos. 17 y 18 de noviembre de 2011 Sede de la UNESCO, París.

UNESCO, (2015). El Futuro del aprendizaje 2 ¿Qué tipo de aprendizaje se necesita en el siglo XXI?. 